\title{
In vitro chondrogenic commitment of human Wharton's jelly stem cells by co-culture with human articular chondrocytes
}

\author{
R. C. Pereira ${ }^{1,2 *}$, A. R. Costa-Pinto ${ }^{1,2}$, A. M. Frias ${ }^{1,2}$, N. M. Neves ${ }^{1,2}$, H. S. Azevedo ${ }^{1,2}$ and R. L. Reis ${ }^{1,2}$ \\ ${ }^{1} 3$ Bs Research Group - Biomaterials, Biodegradables and Biomimetics, University of Minho, Headquarters of the European Institute of \\ Excellence on Tissue Engineering and Regenerative Medicine, AvePark, Guimarães, Portugal \\ ${ }^{2}$ ICVS/3Bs PT Government-associated Laboratory, Braga/Guimarães, Portugal
}

\begin{abstract}
Wharton's jelly stem cells (WJSCs) are a potential source of transplantable stem cells in cartilageregenerative strategies, due to their highly proliferative and multilineage differentiation capacity. We hypothesized that a non-direct co-culture system with human articular chondrocytes (hACs) could enhance the potential chondrogenic phenotype of hWJSCs during the expansion phase compared to those expanded in monoculture conditions. Primary hWJSCs were cultured in the bottom of a multiwell plate separated by a porous transwell membrane insert seeded with hACs. No statistically significant differences in hWJSCs duplication number were observed under either of the culture conditions during the expansion phase. hWJSCs under co-culture conditions show upregulations of collagen type I and II, COMP, TGFB1 and aggrecan, as well as of the main cartilage transcription factor, SOX9, when compared to those cultured in the absence of chondrocytes. Chondrogenic differentiation of hWJSCs, previously expanded in co-culture and monoculture conditions, was evaluated for each cellular passage using the micromass culture model. Cells expanded in co-culture showed higher accumulation of glycosaminoglycans (GAGs) compared to cells in monoculture, and immunohistochemistry for localization of collagen type I revealed a strong detection signal when hWJSCs were expanded under monoculture conditions. In contrast, type II collagen was detected when cells were expanded under co-culture conditions, where numerous round-shaped cell clusters were observed. Using a micromass differentiation model, hWJSCs, previously exposed to soluble factors secreted by hACs, were able to express higher levels of chondrogenic genes with deposition of cartilage extracellular matrix components, suggesting their use as an alternative cell source for treating degenerated cartilage. Copyright (C) 2015 John Wiley \& Sons, Ltd.
\end{abstract}

Received 23 June 2015; Accepted 11 July 2015

Keywords human stem cells; human articular chondrocytes; co-culture; chondrogenic differentiation; cartilage

\section{Introduction}

Cartilage is an avascular musculoskeletal tissue with a low capacity for self-repair. Damage by trauma or disease results in the loss of partial or complete tissue functionality. During the past 15 years, several clinical approaches, such

*Correspondence to: $R$. C. Pereira, Department of Polymer Engineering, 3Bs Research Group - Biomaterials, Biodegradables and Biomimetics, University of Minho, AvePark, Guimarães, Portugal. E-mail: rpereira@dep.uminho.pt as microfracture, arthroscopy and laser abrasion, have been performed for partially restoring function to pathologically altered cartilage (Hunziker, 2002; Risbud and Sittinger, 2002; Cancedda et al., 2003; Mano and Reis, 2007). The autologous chondrocyte implantation (ACI) technique, proposed by Brittberg et al. (1994), is a clear example of a cell-based therapy with excellent clinical results. The use of ACI is associated with a number of limitations, such as morbidity of the surgical procedure, frequent occurrence of periosteal hypertrophy and inefficient cell retention. In addition, it involves lengthy and costly cell isolation and expansion steps of human 
articular chondrocytes (hACs), which have low cellular mitosis and are prone to dedifferentiation when expanded in two dimensions (2D). A potential improvement in ACI procedure is the application of mesenchymal stem cells (MSCs) (Tuan, 2006) instead of hACs in order to minimize additional donor site morbidity. MSCs are attractive candidates for cartilage cell-based therapies because they have the potential to differentiate into the chondrogenic lineage when supplemented with proper differentiation signals (Johnstone et al., 1998; Pittenger et al., 1999; Tuan, 2006; Hwang et al., 2007). Adult stem cells present a limited degree of proliferation, with maintenance of their multipotency capacity of differentiation. Recently, fetal stem cells have attracted increased interest due to their unique features, such as a high capacity to proliferate, maintenance of the self-renewal potential over time and the ability to differentiate towards almost all cell types (Jo et al., 2008; Marcus and Woodbury, 2008). These cells are derived from supportive extra-embryonic structures of fetal origin, such as umbilical cord blood, Wharton's jelly, amniotic fluid, amniotic membrane and placenta, which are routinely discarded after birth. Being extra-embryonic fetal tissues with a large mass volume, these tissues are suitable sources for stem cell isolation. In particular, stem cells derived from the human umbilical cord outer region, known as Wharton's jelly stem cells (WJSCs), have been isolated from the mucoid connective tissue that surrounds the two arteries and the single vein of the umbilical cord. These cells retain many embryonic stem cells (ESCs) and MSC markers in primary cultures at early passages and can be expanded without significant loss of stemness for at least 50 passages (Jo et al., 2008). Recently, various authors have shown that WJSCs present several advantages compared to adult MSCs, such as higher frequency of colony-forming-unit fibroblasts (CFU-Fs) and shorter population doubling time, both critical for scaling-up stem cell cultures (Lu et al., 2006). WJSCs can differentiate into adipogenic, osteogenic, chondrogenic and cardiomyogenic lineages (Wang et al., 2004) and dopaminergic neurons (Fu et al., 2006; Weiss et al., 2006). These cells also express some pluripotent stem cell markers, including Oct-4, Sox-2 and Nanog in cells of porcine origin (Carlin et al., 2006), Oct-4, SSEA4 and c-Kit in cells from equine sources (Hoynowski et al., 2007) and SSEA-1, SSEA-4, Tra-1-60 and Tra-181 in cells from human sources (Jo et al., 2008), suggesting a more immature state than adult MSCs.

Controlling cell differentiation is one of the most challenging aspects of cell-based regenerative therapies, since the mechanisms by which stem cells differentiate can be difficult to recapitulate with current technologies. Coculture systems with relevant cells can be used as valuable tools for probing and manipulating the molecular mechanisms by which stem cells differentiate and could offer therapeutic possibilities in cartilage regeneration strategies. Previously, chondrocytes have been shown to secrete various soluble morphogenic factors able to promote the chondrogenic differentiation of MSCs (Hwang et al., 2007). In this study, we hypothesized that factors secreted by chondrocytes possess chondrogenic-differentiating effects and could enhance chondrogenesis of human Wharton's jelly stem cells (hWJSCs) when co-cultured with human articular chondrocytes (hACs). Particularly, we investigated: (a) whether chondrocytes cultured in a Transwell system could influence the proliferation ratio of hWJSCs during expansion (as illustrated in Figure 1); (b) the relative gene expression of hWJSCs in the presence or absence of hACs; (c) the effect of non-direct co-culture on the ability of hWJSCs to differentiate into the chondrogenic lineage, using the micromass culture model.

Previous work has shown that hWJSCs cultured in three-dimensional (3D) scaffolds in the conditioned medium of hACs cultures presented higher expression of aggregan, SOX9 and collagen type II than those cultured in the absence of hACs medium (Alves da Silva et al., 2015). However, to the best of our knowledge, the effect of non-direct co-culture with hACs on the ability of hWJSCs to undergo chondrogenic differentiation in micromass pellets has not been reported. We believe that considerable efforts will be directed to this area of research, since WJSCs are a potential cell source for musculoskeletal tissue engineering.

\section{Materials and methods}

\subsection{Isolation of $h W J S C s$ and $h A C s$}

Human umbilical cords were collected from mothers who underwent full-term pregnancy, after their consent and approval from the São Marcos Hospital (Braga, Portugal) Ethics Committee.

Briefly, the cord was sectioned into four pieces and the blood vessels were separated from the Wharton's jelly. The collected vessels were washed in phosphate-buffered saline (PBS; pH 7.4, Invitrogen, UK) solution and loops were created with sutures. The vessels were incubated in a $0.50 \mathrm{mg} / \mathrm{ml}$ collagenase type I (Sigma, St. Louis, MO, USA) solution for $18 \mathrm{~h}$ at $37^{\circ} \mathrm{C}$ under slow agitation. After incubation, the digested vessels were diluted with PBS. The solution was collected and centrifuged for $10 \mathrm{~min}$ at $1150 \mathrm{rpm}$. This step was repeated until the solution viscosity was reduced. The vessels were discarded and the cell suspension was incubated with erythrocyte lysis buffer and incubated for $5 \mathrm{~min}$ at room temperature (RT). The cell suspension was centrifuged and the obtained cell pellet dissolved in culture medium. The cells were counted and plated onto a culture flask.

Bioptic material for articular chondrocyte isolation was collected from the femoral condyles of patients undergoing partial knee arthroplasty (three female patients, median age 73 years). All patients signed an informed consent approved by the Ethical Committee of São Marcos Hospital.

Articular cartilage was cleaned of connective tissue and subchondral bone, minced into small fragments and rinsed in fresh PBS, $\mathrm{pH} 7.4$, according to previously published 
A

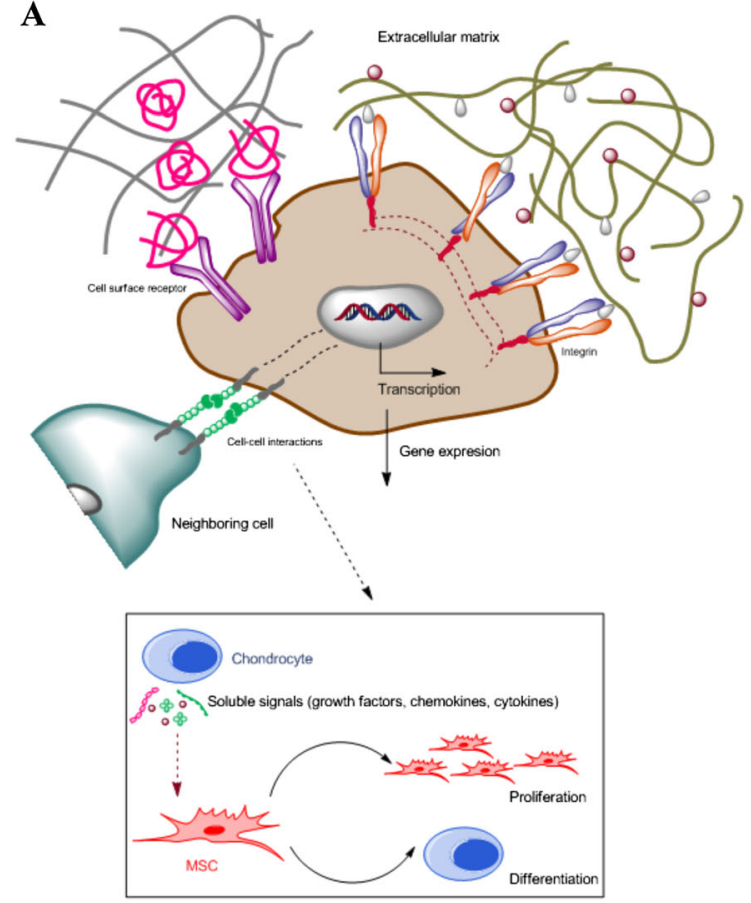

B

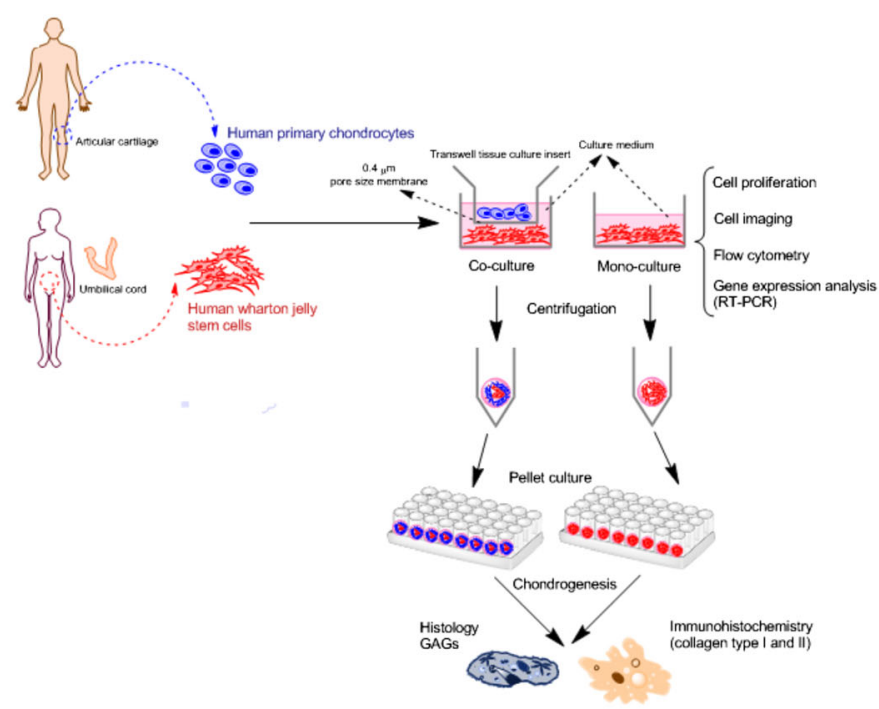

Figure 1. (A) Environmental factors affecting cell behaviour (adapted from Lutolf and Hubbell, 2005). Cells receive multiple signals from their surroundings including neighboring cells. In this study, we investigated whether chondrocyte-secreted morphogenic factors can stimulate the chondrogenic commitment of human Wharton's jelly stem cells (hWJSCs). (B) Experimental design and schematic representation of the culture conditions used in this study

procedures (Pereira et al., 2009). The chondrocytes were individually released by consecutive enzymatic digestion; $400 \mathrm{U} / \mathrm{ml}$ collagenase I, $1000 \mathrm{U} / \mathrm{ml}$ collagenase II (Worthing Biochemical, Lakewood, NJ, USA), 0.25\% trypsin (Invitrogen, UK), $1 \mathrm{mg} / \mathrm{ml}$ hyaluronidase (Sigma, USA) in Dulbecco's modified Eagle's medium (DMEM; Sigma-Aldrich, Germany) with phenol red at $37^{\circ} \mathrm{C}$.

\subsection{Co-culture of hWJSCs with hACs}

For co-culturing hWJSCs with hACs, a transwell system (Corning Inc., Transwell ${ }^{\circledR}$ ) was used where hACs, at a cell density of 18000 cells $/ \mathrm{cm}^{2}$, were plated in the Transwell chambers and placed above the hWJSCs layer, plated at a density of 15000 cells $/ \mathrm{cm}^{2}$. Cells share the same medium, but no direct cell-cell contact is allowed, due to the physical separation of the cells by a polycarbonate membrane with pore size $0.4 \mu \mathrm{m}$, which does not allow cell migration through the membrane (Figure 1B). Cells were cultured in $\alpha$-medium (Sigma, USA) supplemented with 10\% fetal bovine serum (FBS; Biochrom AG, Germany), $5 \mathrm{~mm}$ L-glutamine (Sigma, St. Louis, MO, USA) and $1 \%$ antibiotic-antimicotic mixture (Invitrogen). hACs plated in the inserts were replenished at each enzymatic detachment of hWJSCs at P1. Chondrocytes on the Transwell inserts were trypsinized and processed for cell imaging, staining and PCR (see below, section 2.3.4).

\subsection{Characterization of chondrogenic-stimulated hWJSCs}

\subsubsection{Growth kinetics}

The growth rate (number of cell doublings) of hWJSCs, cultured in the presence or absence of chondrocytes, was calculated with respect to their starting number plated at each passage, $1.5 \times 10^{4} \mathrm{cells} / \mathrm{cm}^{2}$ in the six multiwell tissueculture plates. Number of duplications was plotted vs time to show the effect of culture conditions on cell proliferation. The cells were successively passaged using trypsin-EDTA (Invitrogen Life Technologies, Carlsbad, CA, USA), counted with trypan blue (Invitrogen, UK) staining using a Newbauer chamber. Each time point was assessed $(n=3)$.

\subsubsection{Cell morphology}

hWJSCs cultured on six-well cell-culture plates were observed during the course of culture, using a brightfield microscope (AXIOVERT $40 \mathrm{CFL}$, Germany) equipped with a digital camera (Canon Power Shot G8, Japan). Images at different passages were acquired at $\times 5$ and $\times 10$ magnification.

\subsubsection{Expression of surface markers - flow cytometry}

The phenotype of hWJSCs cultured on polystyrene tissue culture plates (monoculture) and in co-culture with hACs in multiwell inserts was assessed by flow cytometry. Briefly, harvested cells were incubated with fluorescent monoclonal 
antibodies against CD105 (AbD Serotec, UK), CD44 and CD90 (BD Biosciences Pharmingen, USA) for $15 \mathrm{~min}$ at room temperature (RT). The cells were then washed in PBS with $1 \%$ sodium azide (Sigma) and fixed with $2 \%$ formaldehyde. Unlabelled controls were included in every experiment to evaluate non-specific binding. The samples were analysed using a FACScalibur with CellQuest analysis software (both Becton-Dickinson, USA).

\subsubsection{Gene expression - RNA extraction and quantitative reverse transcriptase polymerase chain reaction ( $q R T-P C R)$}

hWJCs co-cultured with hACs and in monoculture conditions were collected at each passage, washed in PBS, immersed in Trizol@ reagent (Invitrogen) and kept at $-80^{\circ} \mathrm{C}$ for subsequent RNA extraction (Pereira et al., 2009). Briefly, the cells were incubated at $4^{\circ} \mathrm{C}$ for $10 \mathrm{~min}$ with chloroform (Sigma) and then centrifuged at $13000 \mathrm{rpm}$ for $15 \mathrm{~min} ; 700 \mu \mathrm{l}$ supernatant were collected and an equivalent volume of isopropanol (Sigma/I-9516) was added. After RNA precipitation (which occurred within $1 \mathrm{~h}$ ), the samples were centrifuged at $13000 \mathrm{rpm}$ and $4^{\circ} \mathrm{C}$ for $15 \mathrm{~min}$. The supernatant was removed and $700 \mu 170 \%$ ethanol was added. The Eppendorf tubes were again centrifuged at $13000 \mathrm{rpm}$ at $4^{\circ} \mathrm{C}$ for $5 \mathrm{~min}$, and the supernatant was removed. The pellet was left to air-dry at RT and at the end was resuspended in $50 \mu \mathrm{l}$ DNase/RNase-free distilled water (Gibco/10977-015). RNA content and integrity was assessed using a ND-1000 spectrophotometer (NanoDrop Technologies, USA). Isolated RNA was transcribed into cDNA using the iScript cDNA synthesis kit (1708891). Relative gene expression quantification was performed by quantitative real-time RT-PCR (qPCR) using a BioRad CFX96 real-time PCR detection system (BioRad Laboratories, CA, USA) and SYBR Green IQ Supermix (Bio-Rad Laboratories). Primer Express software was used to generate the forward and reverse oligonucleotides listed in Table 1 . cDNA ( $2 \mu \mathrm{l}$ in a total volume of $25 \mathrm{ml} /$ reaction) was analysed for the gene of interest and for the reference gene glyceraldehyde-3phosphate dehydrogenase (GAPDH). The expression level of each target gene was calculated using the ${ }^{-2 \Delta 4} C \mathrm{~T}$ method, as described by Livak and Schmittgen (2001). Each sample was analysed three times for the gene of interest.

\subsection{Chondrogenic differentiation of hWJSCs}

\subsubsection{Micromass pellet culture}

The chondrogenic differentiation capacity of hWJSCs, previously exposed to soluble factors secreted by chondrocytes during cell expansion, was investigated using a 3D high-density cell micromass culture model, as described by Johnstone et al. (1998). Briefly, at each passage, cells were trypsinized and $2.5 \times 10^{5}$ cell aliquots were collected in $15 \mathrm{ml}$ conical tubes (Sarstedt, Numbrecht, Germany). The cells were suspended in chondrogenic medium, consisting of serum-free DMEM supplemented with insulin-transferrin-selenium (ITS +1 ; Sigma, St. Louis, MO, USA), $10 \mathrm{ng} / \mathrm{ml}$ transforming growth factor (TGF) $\beta 1,100 \mathrm{~nm}$ dexamathasone, $50 \mu \mathrm{g} / \mathrm{ml}$ ascorbic acid, $2 \mathrm{~mm}$ L-glutamine, $40 \mu \mathrm{g} / \mathrm{ml} \mathrm{L}$-proline and $1 \mathrm{~mm}$ sodium pyruvate. The suspended cells were centrifuged for $2 \mathrm{~min}$ at $1400 \mathrm{rpm}$ to allow the formation of spherical pellets. The pellets $(n=3)$ were cultured in chondrogenic medium for 3 weeks with medium changes every 2 days, and subsequently processed for histological and immunohistochemical analysis.

\subsubsection{Deposition of cartilage extracellular matrix (ECM) - histology and immunohistochemistry}

For histological and immunohistochemical analyses, pellets were fixed in $10 \%$ neutral buffered formalin (formalin, Sigma), washed twice in PBS for $15 \mathrm{~min}$, dehydrated in a graded series of ethanols and then embedded in paraffin. Cross-sections ( $4 \mu \mathrm{m}$ thickness) were cut, dewaxed and stained with safranin $\mathrm{O}$ for detection of sulphated glycosaminoglycan (sGAG). Briefly, the slides were first washed in running tap water, quickly destained with fresh acid ethanol (1\% hydrochloric acid in 70\% ethanol), again washed in running tap water and then immersed in 1:500 fast green (Fluka, cat. no. 44715) for $3 \mathrm{~min}$, after which they were immersed in 1\% acetic acid (Panreac, cat. no. 131008) solution for $30 \mathrm{~s}$ and immersed in $0.1 \%$ safranin O (Fluka, cat. no. 84120) for 4 min. The slides were washed in running tap water, counterstained with haematoxylin, cleared in xylene and mounted. Inserts containing human articular chondrocytes were also processed for safranin $\mathrm{O}$ staining. They were washed twice with PBS, fixed in formalin and stained for GAG detection.

Table 1. Primers used to evaluate gene expression of hWJSCs by qRT-PCR

\begin{tabular}{|c|c|c|}
\hline Gene & Forward & Reverse \\
\hline GAPDH & 5'-ACAGTCAGCCGCATCTTCTT-3' & 5'-ACGACCCAAATCCGTTGACTC-3' \\
\hline Collagen type I & 5'-CATCTCCCCTTCGTTTITGA-3' & 5'-CCAAATCCGATGTTTCTGCT-3' \\
\hline Collagen type II & 5'-GACAATCTGGCTCCCAAC-3' & 5'-ACAGTCTTGCCCCACTTAC-3' \\
\hline Collagen type $X$ & 5'-CCAGGTCTCGATGGTCCTAA-3' & 5'-GTCCTCCAACTCCAGGATCA-3' \\
\hline Sex-determining region Y box 9 (SOX9) & 5'-TACGACTACACCGACCACCA-3' & 5'-TTAGGATCATCTCGGCCATC-3' \\
\hline Cartilage matrix oligomeric protein (COMP) & 5'-CCCACAGACCCTTCCAAGTA-3' & 5'-GGGGACAACTGGAGTGAAAA-3' \\
\hline Transforming growth factor- $\beta$ (TGF $\beta$ ) & 5'-CTCCTCGGAAGACACTCTG-3' & 5'-AGACTGCGCCTGGTAGTTG-3' \\
\hline Aggrecan & 5'-TGAGTCCtTCAAGCCTCCTGT-3' & 5'-TGGTCTGCAGCAGTTGATTC-3' \\
\hline Runt-related transcription factor 2 (Runx2) & 5'-TTCCAGACCAGCAGCACTC-3' & 5'-CAGCGTCAACACCATCATTC-3' \\
\hline
\end{tabular}


For immunohistochemistry examination, slides were dewaxed, washed with PBS solution and the exogenous peroxidase activity quenched with $0.6 \%$ hydrogen peroxide in methanol for $10 \mathrm{~min}$ and blocked with RTU Vectastain ${ }^{\circledR}$ normal horse serum [Vectastain ${ }^{\circledR}$ Universal Elite ABC Kit (Vector, cat. no. VCPK-7200)] for $20 \mathrm{~min}$. The slides were then incubated with rabbit anti-collagen types I (1:200) and II (1:75) primary antibodies (Abcam, Cambridge, MA, USA) overnight at $4^{\circ} \mathrm{C}$ in a humidified atmosphere. A secondary antibody (Vectastain ABC Kit) was applied for $1 \mathrm{~h}$ at RT in a humidified atmosphere, followed by the avidin-biotinylated enzyme complex (Vectastain ABC Kit) and DAB reagent (Vector Laboratories, cat. no. VCSK-4100), then the slides were counterstained with haematoxylin and mounted. Slides stained without the addition of the primary antibody served as negative controls. Native human articular cartilage was used as a positive control for collagens type I and II.

\subsection{Statistical analysis}

The number of cell duplications and relative gene expression quantifications are expressed as mean \pm standard deviation (SD), with $n=3$ for each culture condition. The statistical significance of differences was determined using Student's t-test multiple comparison procedure at a confidence level of $95 \%(p<0.05)$.

\section{Results}

\subsection{Characterization of hACs during co-culture}

During the co-culture experiments, chondrocytes were seeded on Transwell inserts and stained at each cellular passage with Alcian blue,Toluidine blue or safranin $\mathrm{O}$ to detect the presence of cartilage glycosaminoglycans (GAGs). Multiwell inserts without chondrocytes were also stained and used as controls, to confirm the absence of possible staining artifacts. Membrane inserts did not colour when cells were not present (data not shown). On the contrary, hACs cultured for 1 week were positive for the presence of GAGs (Figure 2A). Imaging of hACs during in vitro co-culture showed that the cells maintained their characteristic polygonal morphology with full colonization of the surface (Figure 2B). The levels of several transcripts of chondrocytes were assessed by means of PCR analysis. Amplified products were resolved on 1\% agarose gels (Figure 2C). Collagen types I and II and aggrecan were present in small amounts. Cartilagespecific transcription factors SOX9, COMP and TGF 1 genes were also detected. These results indicate that hACs were able to maintain their inherent chondrogenic phenotype.

\subsection{Characterization of chondrogenic-stimulated hWJSCs}

Calculation of the duplication number vs time during the total period of culture allowed assessment of the proliferation capacity of hWJSCs, either in co-culture with chondrocytes or in monoculture (Figure 3A). Cellular duplication was constant for the whole culture time. Similar duplication number values were observed for both culture conditions, with no statistically significant differences. Possible changes in cell morphology during expansion were also monitored (Figure 3B). Either under monoculture or co-culture conditions, hWJSCs maintained their characteristic long-shape morphology and colonized the entire plastic surface over the time of
A

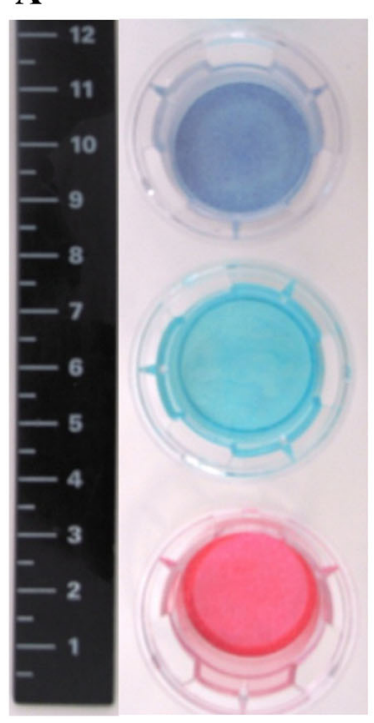

B

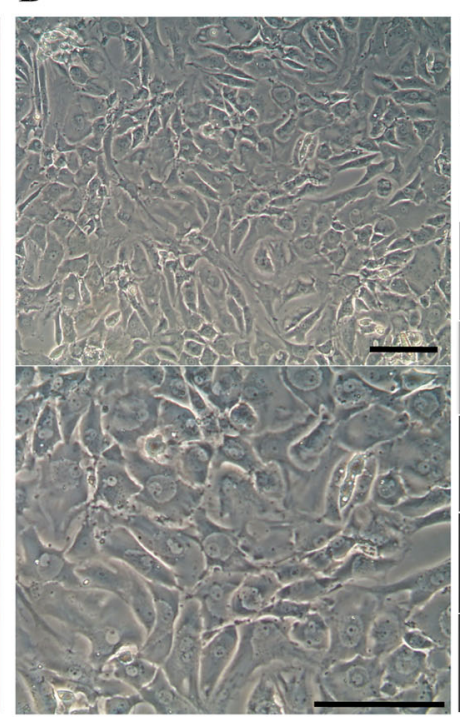

C

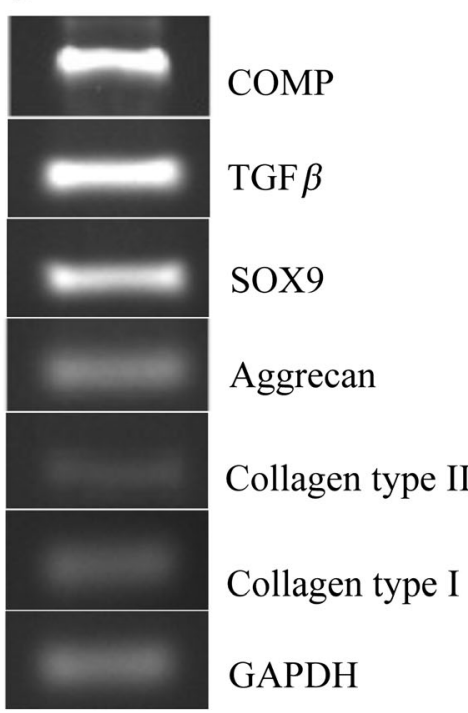

Figure 2. Characterization of human articular chondrocytes (hACs) after 1 week in co-culture with hWJSCs. (A) Toluidine blue, Alcian blue and safranin $\mathrm{O}$ staining (from top to bottom) of hACs cultured on multiwell inserts. (B) Light microscopy images showing chondrocyte morphology in 2D culture; scale bar $=100 \mu \mathrm{m}$; (C) Real-time RT-PCR analysis of chondrogenic marker genes confirming that chondrocytes maintain their phenotype during the culture time. DNA fragments and products of real-time RT-PCR for each gene were set in agarose gel 
A

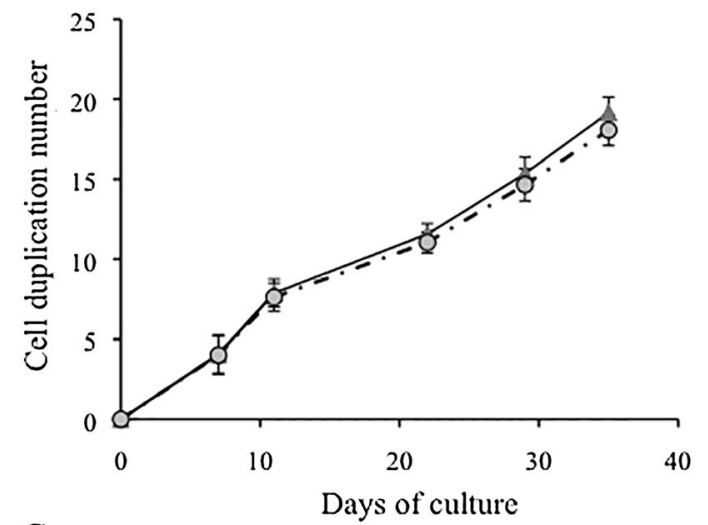

C
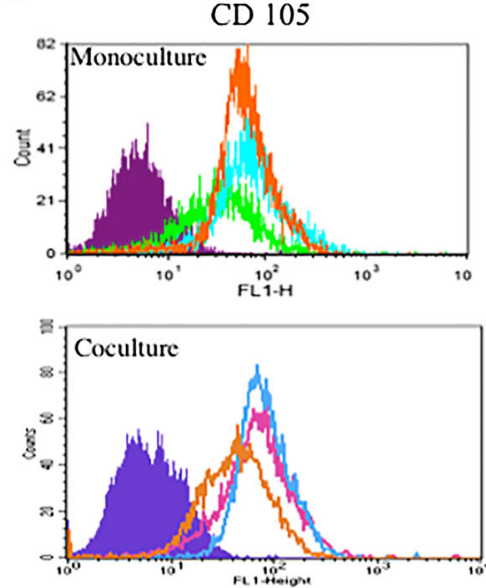

B

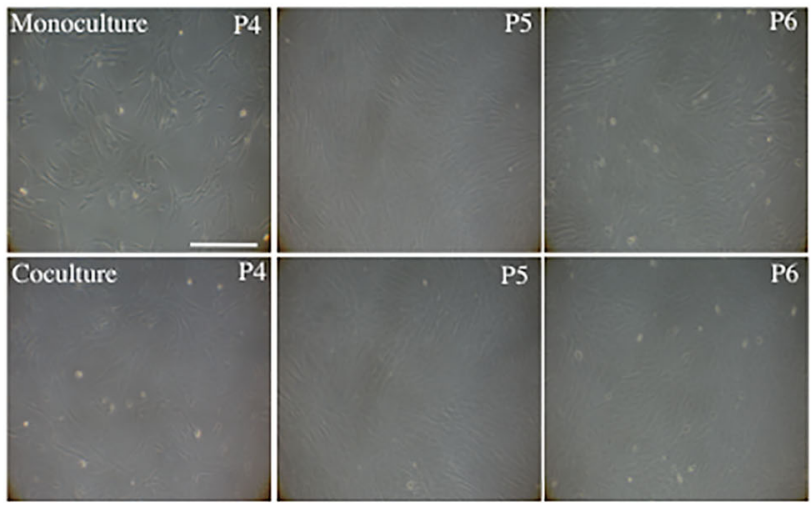

CD 73
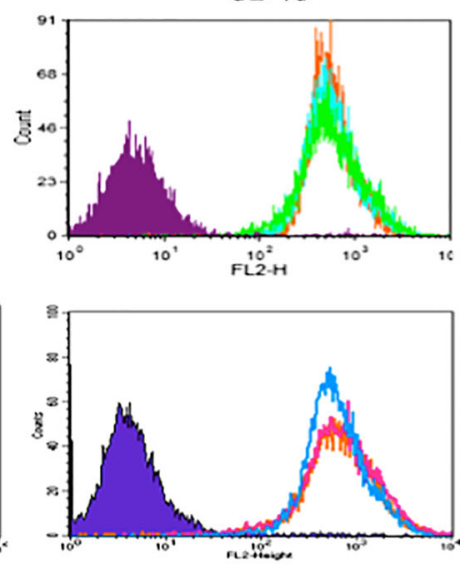
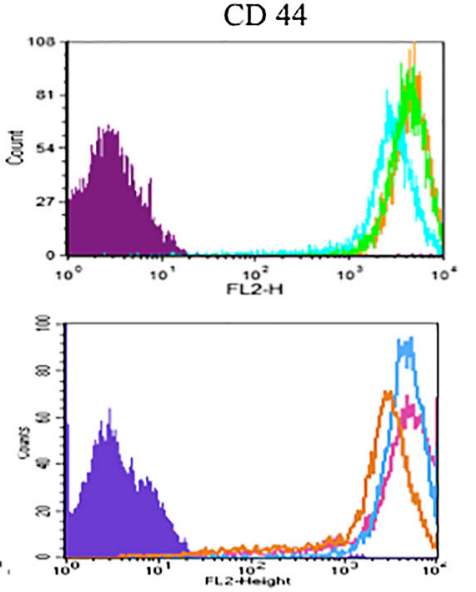

P6

P5

P4

P6

P5

P4

Figure 3. Characterization of chondrogenic-stimulated hWJSCs. (A) Doubling numbers during the expansion phase: growth kinetics plotted as number of cell duplications vs time of culture (proliferation); solid line, hWJSCs co-cultured with hACs; dashed line, hWJSCs in monoculture. (B) Optical microscopy images of hWJSCs in monoculture and co-culture conditions during the expansion phase at passages (P) P4, P5 and P6; scale bar $=100 \mu \mathrm{m}$. (C) Flow-cytometry analysis of CD105, CD73 and CD44 expression on hWJSCs at different passages after co-culture with hACs and in monoculture conditions

culture. Analysis of specific clusters of differentiation (CD) on the surface of hWJSCs during its expansion, under both culture conditions, was assessed by flow cytometry (Figure 3C). Cultured cells were analysed for the presence of mesenchymal stem cell markers (CD44, CD73 and CD105), expression of which was > 95\% and no differences were observed for the different cell passages.

\subsection{Expression profile of chondrocyte-specific genes by hWJSCs}

To assess variations in gene expression by hWJSCs, either in co-culture or in monoculture, $\mathrm{QPCR}$ was performed to quantify the expression profile of several genes by these cells during the expansion phase; GAPDH was chosen as the reference housekeeping gene. Relative gene expression was quantified by normalizing gene values with those expressed by hWJSCs in monoculture (Figure 4). We observed an upregulation of gene expression for all analysed genes when hWJSCs were co-cultured with chondrocytes.
Collagen type I presented the highest relative expression, four-fold at passage 3 . With further passages during cell expansion, the level of collagen type I expression presented a continuous decrease of fold induction. The opposite tendency of fold expression could be observed for type II collagen, which augmented with cell expansion. A drastic reduction of aggrecan expression level was observed from P3 to P4 but then increased until P6. A slight reduction of Sox 9 relative expression was observed with cellular passage. Upregulation of TGF $\beta 1$ was observed for hWJSCs co-cultured with chondrocytes compared to those expanded alone. Relative induction fold values of this gene increased gradually during culture. Cartilage oligomeric matrix protein (COMP) relative expression values tended to increase, especially for the latest stages of cell expansion; this tendency was mainly observed in the first three cell passages. Type $\mathrm{X}$ collagen (a hypertrophic chondrocyte marker) and Runx2 (pre-osteogenic gene marker) relative expression values showed analogous behaviour. A decrease in the first two passages was observed, with further low expression until the sixth passage. 
A

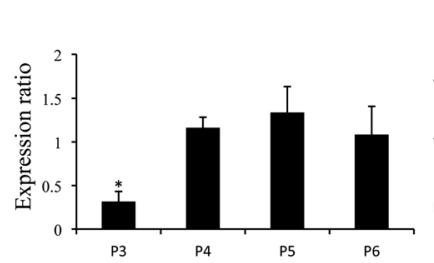

$\mathrm{E}$

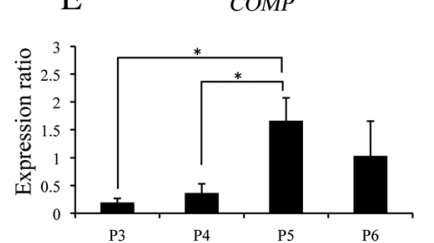

B

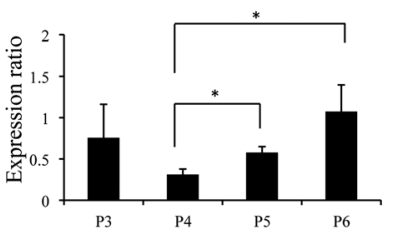

$\mathrm{F}$

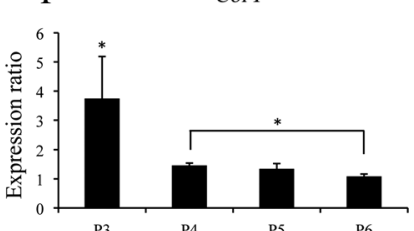

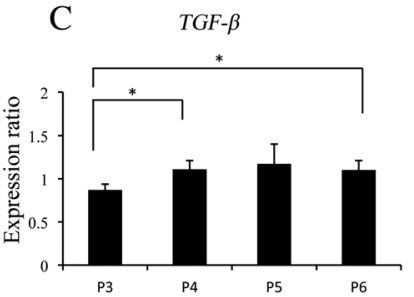
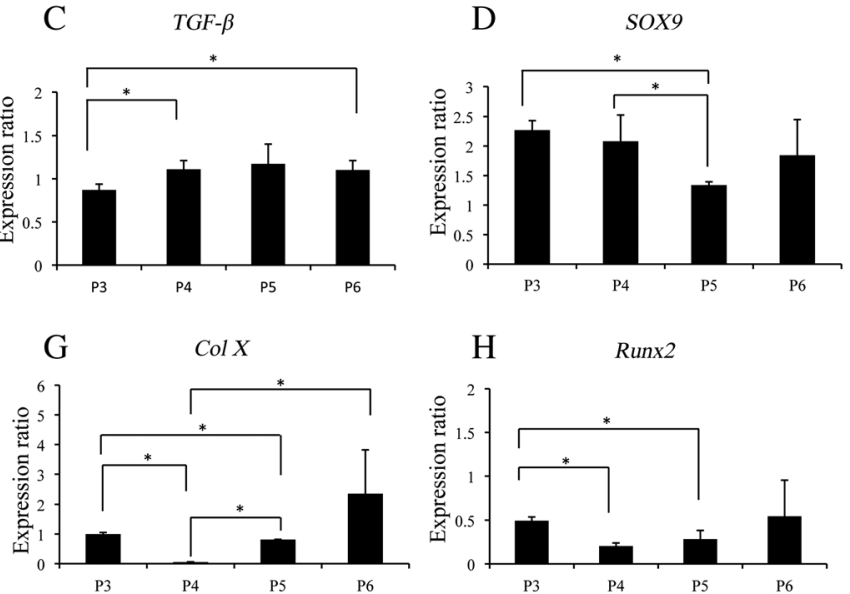

Figure 4. Expression profile of chondrocyte-specific genes (A-E), collagen type I (F), collagen type X (G) and Runx2 (H) by hWJSCs during the expansion phase at different passages. Level of mRNA transcripts fold induction of hWJSCs co-cultured with chondrocytes was normalized to values achieved form hWJSCs cultured alone during the expansion phase. Statistical analysis was performed through the various passages $(p<0.05)$

\subsection{Chondrogenic differentiation of hWJSCs in micromass pellet culture model}

\subsubsection{Deposition of glycosaminoglycans}

Histological analysis of micromass pellets of hWJSCs, previously expanded under co-culture and monoculture conditions, was performed using safranin O staining, which detects cartilage glycosaminoglycans (Figure 5). This analysis showed a non-homogeneous distribution of the cells within micromass pellets. The pellets displayed positive staining for safranin $\mathrm{O}$ at each cellular passage for both culture conditions. Despite this positive staining, hWJSCs cultured in the absence of chondrocytes presented a more fibroblast-like morphology than those expanded in co-culture.

\subsubsection{Deposition of collagen types I and II}

The results from immunohistochemical localization of collagen types I and II can be appreciated in Figure 6. A more positive staining of type I collagen was detected in the micromass pellets obtained with cells expanded in monoculture than those expanded in co-culture (Figure 6). A significant detection of collagen type II in pellets formed with co-cultured hWJSCs was observed.

\section{Discussion}

One of the difficulties of engineering and repairing cartilage is the lack of a sufficient number of cells when using autologous chondrocytes. In recent years, this field of research has shown an increasing interest in using alternative cell sources and a valid culture systems (Sekiya et al., 2002; Tuan, 2006).

Mesenchymal stem cells (MSCs) found in several tissues, such as bone marrow (Bianco et al., 2001), adipose tissue (Zuk et al., 2002) and umbilical cord (Troyer and Weiss, 2008), have demonstrated a multilineage potential and have been considered a potential alternative to autologous chondrocytes. Barry et al. (2001) showed the chondrogenic differentiation ability of MSCs from bone marrow with the potential risk of hypertrophy induction. Currently, adipose stem cells are an abundant source of adult stem cells, but their chondrogenic potential is known to be inferior to those from bone marrow (Huang et al., 2005).

Like human umbilical cord stem cells (Bailey et al., 2007; Wang and Detamore, 2009; Wang et al., 2009a, 2009b), we suggest that hWJSCs could be a useful cell source for cartilage regeneration strategies. This cell type combines most of the ESC and adult MSCs 'stemness' markers for long expansion periods (Jo et al., 2008), showing the ability to differentiate along mesenchymal lineages (Wang et al., 2004; Can and Karahuseyinoglu, 2007). Harvested from umbilical cords, hWJSCs are easily accessible, presenting high levels of proliferation during in vitro expansion and making it possible to obtain a plentiful amount of cells in a short period of time (Baksh et al., 2007; Can and Karahuseyinoglu, 2007).

Cartilage tissue engineering success depends not only on biomaterial selection, but also on the development of efficient approaches to control cell differentiation and neo-tissue assembly. In this perspective, chondrocytes have been employed in several studies using co-culture systems, with direct or non-direct cell contact, to achieve either chondrogenic or osteogenic differentiation of mesenchymal stem cells (Gerstenfeld et al., 2002, 2003; Hwang et al., 2007).

The use of hACs in a non-direct co-culture system with hWJSCs (Alves da Silva et al., 2015) has not been fully explored, and the increased interest in this cell type in cartilage tissue engineering led us to propose this study. Co-culture of hWJSCs with hACs using $\alpha$-medium supplemented with 10\% FBS for almost 36 days did not show statistically significant differences in the proliferation 
A
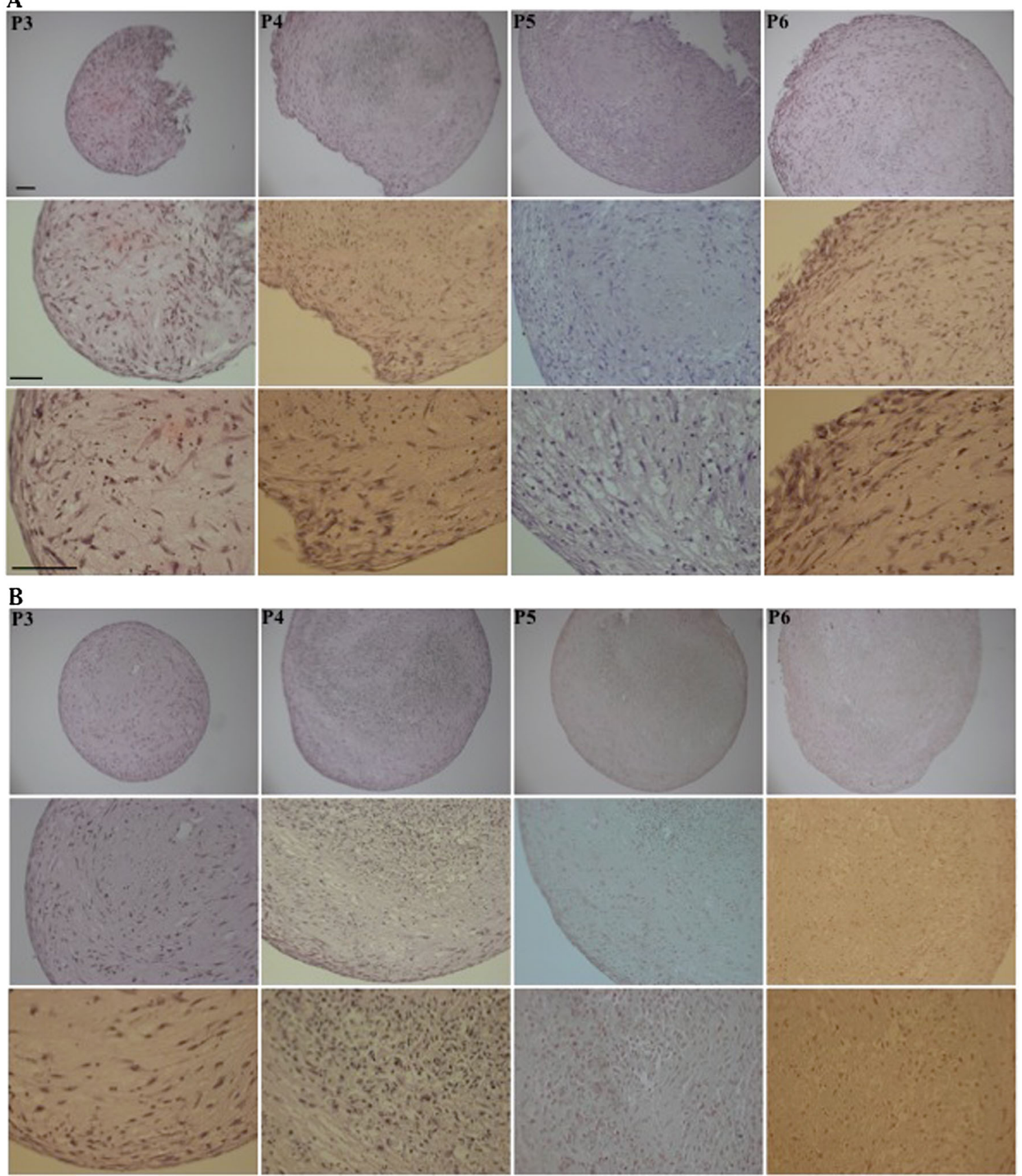

Figure 5. Histological staining of 3D micromass pellet cross-sections of hWJSCs after being expanded in monoculture (A) or in co-culture with hACs (B), stained with safranin $\mathrm{O}$, which detects the presence of sulphated glycosaminoglycans; scale bar $=100 \mu \mathrm{m}$

ratio, compared to those cultured under the same conditions but in the absence of chondrocytes (Figure 3). This result was not in agreement with data found in previous studies, where soluble factors released from chondrocytes enhanced the survival and proliferation of mesenchymal cells (Solursh, 1975; Hwang et al., 2007, 2008), but none of these studies used hWJSCs. Under both conditions, hWJSCs achieved ca. 20 duplications (corresponding to a 64-fold increase of the initial cell number) in about 36 days of culture. It is well known that chondrocytes expanded ex vivo undergo phenotype changes, with the different expression of several chondrogenic gene markers depending on expansion time (von der Mark et al., 1977; Malpeli et al., 2004). For this reason, each time
hWJSCs were counted and replated in the polystyrene culture plates, on average every 8 days, hACs were also detached by enzymatic digestion with trypsin. Fresh hACs at P1 were replated in the insert wells. This was performed to avoid further expansion of hACs and thus prevent their possible dedifferentiation (Malpeli et al., 2004). As shown in Figure 2A, hACs seeded on Transwell inserts were macroscopically positive for Alcian blue, toluidine blue and safranin $\mathrm{O}$ staining, demonstrating their ability to accumulate cartilage GAGs. The low level of collagen type I expression indicated a low cell proliferation ratio, characteristic of the chondrogenic phenotype (Malpeli et al., 2004). When plated in monolayer (2D surfaces), chondrocytes hardly preserved the expression of 


\section{Chondrogenic commitment of human Wharton's jelly stem cells}
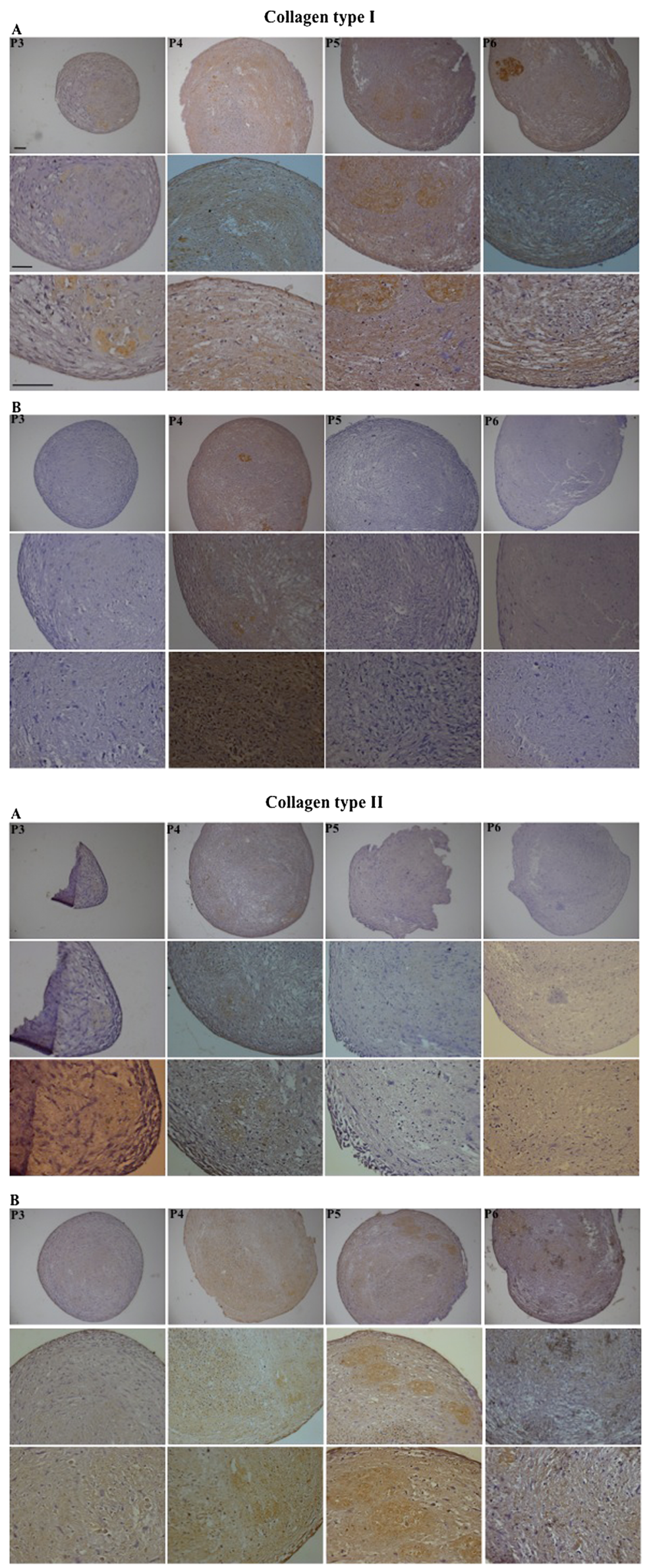

Figure 6. Immunohistochemical detection of collagen types I and II in micromass pellets of hWJSCs after expansion in monoculture (A) and in co-culture with hACs (B); scale bar $=100 \mu \mathrm{m}$ 
collagen type II (Malpeli et al., 2004). This behaviour was also observed in our study, confirmed by the faint line observed for the amplified DNA fragment. Nevertheless, we observed that hACs at P1 were able to express other typical cartilage marker genes, such as aggrecan, COMP and TGF $\beta 1$, as well as the cartilage master transcription factor SOX9, an important protein implicated in the maintenance of the chondrogenic phenotype in articular chondrocytes (Malpeli et al., 2004). Based on these results, we assumed that human articular chondrocytes plated in the multi-well inserts maintained the majority of their chondrogenic phenotype characteristics.

The defining characteristics of MSCs include their ability to self-renew and to express specific markers, such as CD73, CD90 and CD105, on their surface (Delorme et al., 2008; Jin et al., 2009). CD105, a 180 kDa integral membrane glycoprotein known as endoglin, is a receptor for TGF $\beta 1$ and TGF $\beta 3$ and modulates TGF $\beta$ signalling by interacting with related molecules, such as TGF $\beta 1$, TGF $\beta 3$, BMP-2 and BMP-7. It is speculated that these members of the TFG $\beta$ superfamily are mediators of cell proliferation and differentiation and play regulatory roles in cartilage and bone formation. The disappearance of CD105 antigen during osteogenesis suggests that this protein, like others in the TFG $\beta$ superfamily, may be involved in the regulation of osteogenesis. Recent studies reported that members of the TGF $\beta$ family control the differentiation of MSCs (Haynesworth et al., 1992; Jakob et al., 2001). However, whether CD105 plays a functional role during the process of stem cell differentiation has not yet been clarified. Therefore, we evaluated the potential effect of chondrocyte soluble factors over the expression profile of MSCs markers (CD44, CD73 and CD105) on hWJSCs co-cultured with hACs for several passages (Figure $3 \mathrm{C}$ ). No significant differences were observed at the phenotypic level under co-culture conditions.

Cartilage master transcription factor SOX9 (Lefebvre et al., 1998; Sekiya et al., 2002; Malpeli et al., 2004) (Figure 4), was upregulated in hWJSCs in co-culture conditions relative to those cultured alone, presenting at P3 and P4 the highest values of relative fold induction, 2.4 and 2.2, respectively. With further cell expansion, a minor decrease of relative fold until P6 was observed. Relative fold induction values of TGF $\beta 1$ gradually increased from P3 up to P5 (0.9-1.2), presenting a plane behaviour for the next passage (1.1-fold). Cartilage oligomeric matrix protein (COMP) has an important role in cartilage phenotype maintenance (Zaucke et al., 2001). COMP relative expression shows a slow increase from P3 to P4 (0.2-0.4 in that order) with a subsequent significant enhancement up 1.6- and 1.1-fold, respectively, at P5 and P6. On the other hand, collagen type I expression presented its higher value of expression, four-fold, at P3. With further cell expansion, collagen type I expression presented low values, it being possible to perceive a statistically significant continuous reduction tendency of expression until the end of the study. Collagen type $\mathrm{X}$ and Runx2 relative expression presented a similar trend. A significant decrease from P3 to P4, followed by a slight increase at P5, was observed.
Cells cultured at P6 presented higher gene expression values, but these were not statistically significant. The gene expression data suggest that hACs can be responsible for a possible chondrogenic commitment of hWJSCs when expanded in 2D monolayer surface. These results suggest that co-culture with hACs provides anabolic soluble factors to hWJSCs towards the chondrogenic phenotype, without the need to add exogenous growth factors. It was not the aim of the present study to determine which factors could lead to this commitment. Further research should focus on the identification of important factors that might be responsible for driving stem cells into the chondrogenic phenotype. Using different stem cell types, Hwang et al. (2008) and Kim et al. (2007) suggested that TGF $\beta$ and cytokine-like 1 (Cytl1) could modulate a chondrogenic phenotype in human embryonic stem cells and mesenchymal stem cells, respectively (Kim et al., 2007). Cytl1 protein, oversecreted by chondrocytes, showed the ability to promote the chondrogenic differentiation of mesenchymal cells.

The use of chondrocytes collected and isolated from the femoral condyles of patients undergoing partial knee arthroplasty is a limitation of the current study. It is remarkably difficult to obtain healthy hACs for cartilage research. However, knee arthropasties, commonly performed worldwide, can provide relatively healthy cartilage tissue which, after digestion/isolation, allows a source of primary adult articular chondrocytes to be obtained for cartilage research and therapy. Our data show that chondrocytes obtained from non-totally healthy cartilage biopsies could promote the chondrogenic differentiation of hWJSCs by committing these cells to the chondrogenic phenotype during 2D expansion.

Safranin O staining (Figure 5) showed the presence of glycosaminoglycans, characteristic components of cartilage ECM or in cells exhibiting a chondrogenic phenotype, due to a successful chrondrogenic differentiation process. Figure 5A shows that, in expansion in monoculture conditions, hWJSCs display a fibroblast-like morphology, which can be also observed in the external region of hWJSC micromass pellets expanded in co-culture (P4-P6). Nevertheless, under this culture condition, aggregates of cells with round shape and more positive safranin $\mathrm{O}$ staining can be observed at the pellet core.

Immunohistochemical localization of collagen type I and II in the micromass pellets maintained in culture for 3 weeks was performed to assess the chondrogenic phenotype level accomplished by hWJSCs expanded in mono- and co-culture conditions. The presence of collagen type I was detected in cells expanded in monoculture (Figure 6A). From the beginning (P3), several positively stained cellular regions were observed, demonstrating incomplete/reduced chondrogenic differentiation of the cells. After expansion under the co-culture condition, micromass pellets of hWJSCs showed a minimal detection of this protein.

Collagen type II is one of the main cartilage ECM proteins. Under both conditions, hWJSCs were positive for collagen type II. Differences could, however, be observed 
when cells were expanded under different conditions. Looking in detail, several cell nodules with round shape, stained positive for this cartilage ECM protein, were observed in the micromass pellets made of hWJSCs expanded in co-culture at all passages. This collagen type II positive staining on the spherical cellular aggregates is not observed in hWJSCs cultured alone, being markedly weak within the pellet.

Collagen types I and II detection (Figure 6) corroborated the results of safranin $\mathrm{O}$ staining, showing greater detection of cartilage ECM components in hWJSCs expanded in co-culture with chrondrocytes compared to those from hWJSCs cultured alone. Soluble factors released from hACs during co-culture may stimulate hWJSCs to produce cartilage ECM components during micromass pellet culture.

These results, in addition to the real-time RT-PCR data showing positive modulation of collagens type I and II, aggrecan, Sox9, COMP and TGF $\beta 1$, as well as low values for collagen type $\mathrm{X}$ and Runx2, tend to support our initial hypothesis of the ability of chondrocytes to secrete soluble active growth factors with a capacity to influence hWJSCs phenotype in co-culture. In ascertaining which are the metabolic soluble factors and their possible mechanisms of action on the chondrogenic commitment of hWJSCs, the results of this study indicate the potential of using stem cells and their co-culture with articular chondrocytes to obtain cells in cartilage repair strategies.

\section{Conclusions}

Our study suggests that in vitro expansion of hWJSCs in monolayer and in a non-direct co-culture with human articular chondrocytes can be used as a strategy to commit a stem cell source to the chondrogenic phenotype. Although promising results were obtained during in vitro expansion and using the micromass pellet culturing model, corroboration should be done in an in vivo environment to demonstrate that the proposed hypothesis is sufficient to induce a stable phenotype.

\section{Conflict of interest}

The authors have declared that there is no conflict of interest.

\section{Acknowledgements}

This work was supported by the European Union-funded Network of Excellence 'EXPERTISSUES' (Grant No. NMP3-CT-2004500283). A.R.C.-P. and A.M.F. thank the Portuguese Foundation for Science and Technology for post-doctoral fellowships SFRH/ BPD/90332/2012 and SFRH/BPD/45206/2008, respectively. The authors are grateful to patients from the Hospital de S. Marcos (Braga, Portugal) for the donation of the biological samples, and to the medical staff for their collaboration and support.

\section{References}

Alves da Silva ML, Costa-Pinto AR, Martins A et al. 2015; Conditioned medium as a strategy for human stem cells chondrogenic differentiation. J Tissue Eng Regen Med 9(6): 714-723.

Bailey MM, Wang L, Bode CJ, et al. 2007; A comparison of human umbilical cord matrix stem cells and temporomandibular joint condylar chondrocytes for tissue engineering temporomandibular joint condylar cartilage. Tissue Eng 13: 2003-2010.

Baksh D, Yao R, Tuan RS 2007; Comparison of proliferative and multilineage differentiation potential of human mesenchymal stem cells derived from umbilical cord and bone marrow. Stem Cells 25: 1384-1392.

Barry F, Boynton RE, Liu BS, et al. 2001; Chondrogenic differentiation of mesenchymal stem cells from bone marrow: differentiation-dependent gene expression of matrix components. Exp Cell Res 268: 189-200.

Bianco P, Riminucci M, Gronthos S, et al. 2001; Bone marrow stromal stem cells: nature, biology, and potential applications. Stem Cells 19: 180-192.

Brittberg M, Lindahl A, Nilsson A, et al. 1994; Treatment of deep cartilage defects in the knee with autologous chondrocyte transplantation. N Engl J Med 331: 889-895.

Can A, Karahuseyinoglu S 2007; Concise review: human umbilical cord stroma with regard to the source of fetus-derived stem cells. Stem Cells 25: 2886-2895.
Cancedda R, Dozin B, Giannoni P, et al. 2003; Tissue engineering and cell therapy of cartilage and bone. Matrix Biol 22(1): 81-91. Carlin R, Davis D, Weiss M et al. 2006; Expression of early transcription factors Oct-4, Sox- 2 and Nanog by porcine umbilical cord (PUC) matrix cells. Reprod Biol Endocrinol 4: 8. DOI:10.1186/1477-7827-4-8.

Delorme B, Ringe J, Gallay N, et al. 2008; Specific plasma membrane protein phenotype of culture-amplified and native human bone marrow mesenchymal stem cells. Blood 111: 2631-2635.

Fu YS, Cheng YC, Lin MYA, et al. 2006; Conversion of human umbilical cord mesenchymal stem cells in Wharton's jelly to dopaminergic neurons in vitro: potential therapeutic application for parkinsonism. Stem Cells 24: 115-124.

Gerstenfeld LC, Barnes GL, Shea CM, et al. 2003; Osteogenic differentiation is selectively promoted by morphogenetic signals from chondrocytes and synergized by a nutrient-rich growth environment. Conn Tissue Res 44: 85-91.

Gerstenfeld LC, Cruceta J, Shea CM, et al 2002; Chondrocytes provide morphogenic signals that selectively induce osteogenic differentiation of mesenchymal stem cells. $J$ Bone Miner Res 17: 221-230.

Haynesworth SE, Baber MA, Caplan AI 1992; Cell surface antigens on human marrowderived mesenchymal cells are detected by monoclonal antibodies. Bone 13: 69-80.
Hoynowski SM, Fry MM, Gardner BM, et al. 2007; Characterization and differentiation of equine umbilical cord-derived matrix cells. Biochem Biophys Res Commun 362: 347-353.

Huang JI, Kazmi N, Durbhakula MM, et al. 2005; Chondrogenic potential of progenitor cells derived from human bone marrow and adipose tissue: a patientmatched comparison. J Orthop Res 23: 1383-1389.

Hunziker EB 2002; Articular cartilage repair: basic science and clinical progress. A review of the current status and prospects. Osteoarthr Cartilage 10: 432-463.

Hwang NS, Varghese S, Elisseeff J. 2008; Derivation of chondrogenically-committed cells from human embryonic cells for cartilage tissue regeneration. Plos One 3(6): e2498. DOI:10.1371/journal.pone.0002498.

Hwang NS, Varghese S, Puleo C, et al. 2007; Morphogenetic signals from chondrocytes promote chondrogenic and osteogenic differentiation of mesenchymal stem cells. $J$ Cell Physiol 212: 281-284.

Jakob M, Demarteau O, Schafer D, et al. 2001; Specific growth factors during the expansion and redifferentiation of adult human articular chondrocytes enhance chondrogenesis and cartilaginous tissue formation in vitro. J Cell Biochem 81: 368-377.

Jin HJ, Park SK, Oh W, et al. 2009; Down-regulation of CD105 is associated with multilineage differentiation in human umbilical 
cord blood-derived mesenchymal stem cells. Biochem Biophys Res Commun 381: 676-681.

Jo CH, Kim OS, Park EY, et al. 2008; Fetal mesenchymal stem cells derived from human umbilical cord sustain primitive characteristics during extensive expansion. Cell Tissue Res 334: 423-433.

Johnstone B, Hering TM, Caplan AI, et al. 1998; In vitro chondrogenesis of bone marrow-derived mesenchymal progenitor cells. Exp Cell Res 238: 265-272.

Kim JS, Ryoo ZY, Chun JS 2007; Cytokinelike 1 (CYTL1) regulates the chondrogenesis of mesenchymal cells. J Biol Chem 282: 29359-29367.

Lefebvre V, Li P, de Crombrugghe B 1998; A new long form of Sox5 (L-Sox5), Sox6 and Sox9 are coexpressed in chondrogenesis and cooperatively activate the type II collagen gene. EMBO J 17: 5718-5733.

Livak KJ, Schmittgen TD 2001; Analysis of relative gene expression data using realtime quantitative PCR and the ${ }^{2-4 A} \mathrm{C} \backslash$ method. Methods 25: 402-408.

Lu LL, Liu YJ, Yang SG, et al. 2006; Isolation and characterization of human umbilical cord mesenchymal stem cells with hematopoiesis - supportive function and other potentials. Haematologica 91: 1017-1026.

Lutolf MP, Hubbell JA 2005; Synthetic biomaterials as instructive extracellular microenvironments for morphogenesis in tissue engineering. Nat Biotechnol 23: 47-55.

Malpeli M, Randazzo N, Cancedda R, et al. 2004; Serum-free growth medium sustains commitment of human articular chondrocyte through maintenance of Sox9 expression. Tissue Eng 10: 145-155.
Mano JF, Reis RL 2007; Osteochondral defects: present situation and tissue engineering approaches. $J$ Tissue Eng Regen Med 1: 261-273.

Marcus AJ, Woodbury D 2008; Fetal stem cells from extra-embryonic tissues: do not discard. J Cell Mol Med 12: 730-742.

Pereira RC, Scaranari M, Castagnola P, et al. 2009; Novel injectable gel (system) as a vehicle for human articular chondrocytes in cartilage tissue regeneration. $J$ Tissue Eng Regen Med 3: 97-106.

Pittenger MF, Mackay AM, Beck SC, et al. 1999; Multilineage potential of adult human mesenchymal stem cells. Science 284: 143-147.

Risbud MV, Sittinger M 2002; Tissue engineering: advances in in vitro cartilage generation. Trends Biotechnol 20: 351-356.

Sekiya I, Vuoristo JT, Larson BL, et al. 2002; In vitro cartilage formation by human adult stem cells from bone marrow stroma defines the sequence of cellular and molecular events during chondrogenesis. Proc Natl Acad Sci USA 99: 4397-4402.

Solursh MRR 1975; The enhancement of in vitro survival and chondrogenesis of limb bud cells by cartilage conditioned medium. Dev Biol 44: 278-287.

Troyer DL, Weiss ML 2008; Concise review: Wharton's jelly-derived cells are a primitive stromal cell population. Stem Cells 26: 591-599.

Tuan RS 2006; Stemming cartilage degeneration: Adult mesenchymal stem cells as a cell source for articular cartilage tissue engineering. Arthritis Rheumatism 54: 3075-3078. von der Mark KGV, von der Mark H, Muller P 1977; Relationship between cell shape and type of collagen synthesised as chondrocytes lose their cartilage phenotype in culture. $\mathrm{Na}$ ture 267: 531-532

Wang HS, Hung SC, Peng ST, et al. 2004; Mesenchymal stem cells in the Wharton's jelly of the human umbilical cord. Stem Cells 22: 1330-1337.

Wang LM, Detamore MS 2009; Insulin-like growth factor-I improves chondrogenesis of predifferentiated human umbilical cord mesenchymal stromal cells. $J$ Orthop Res 27: 1109-1115.

Wang LM, Seshareddy K, Weiss ML, et al. 2009a; Effect of initial seeding density on human umbilical cord mesenchymal stromal cells for fibrocartilage tissue engineering. Tissue Eng A 15: 1009-1017.

Wang LM, Tran I, Seshareddy K, et al. 2009b; A comparison of human bone marrowderived mesenchymal stem cells and human umbilical cord-derived mesenchymal stromal cells for cartilage tissue engineering. Tissue Eng A 15: 2259-2266.

Weiss ML, Medicetty S, Bledsoe AR, et al. 2006; Human umbilical cord matrix stem cells: preliminary characterization and effect of transplantation in a rodent model of Parkinson's disease. Stem Cells 24: 781-792.

Zaucke F, Dinser R, Maurer P, et al. 2001; Cartilage oligomeric matrix protein (COMP) and collagen IX are sensitive markers for the differentiation state of articular primary chondrocytes. Biochemical $J$ 358: 17-24.

Zuk PA, Zhu M, Ashjian P, et al. 2002; Human adipose tissue is a source of multipotent stem cells. Mol Biol Cell 13: 4279-4295. 